\title{
Programa de intervención educativa para promover el cambio de actitud hacia la actividad física y la mejora del estilo de vida de los estudiantes de Tecnología Médica en una universidad privada
}

\author{
Cristhian Santiago Bazán 1,a,b
}

\section{RESUMEN}

Objetivo: Determinar la influencia de un programa de intervención educativa para promover el cambio de actitud hacia la actividad física y la mejora del estilo de vida de los estudiantes de Tecnología Médica de una universidad privada.

Materiales y métodos: Estudio cuantitativo, de tipo cuasi experimental, de corte longitudinal, prospectivo. La muestra de estudio incluyó a 94 estudiantes, divididos en dos grupos: A (grupo experimental) y B (grupo control), el cual fue creado y diseñado por el autor, tuvo una duración de 12 sesiones en el semestre académico. 2016-II; los instrumentos empleado fueron el test de Dosel que midió la actitud hacia la actividad física, para medir los estilos de vida saludable se aplicó un cuestionario de 30 preguntas cuya confiabilidad (Alfa de Crombach) alcanzó el valor de 0.95, considerándose una alta fiabilidad. Se utilizó la Prueba T Student, el cual afirmó que existe diferencia significativa entre los promedios del pretest y el post test.

Resultados: El programa de intervención educativa influye en el indicador: importancia percibida $p=0.000$, actitud hacia el indicador: actividad práctica $\mathrm{p}=0.000$. En cuanto a las dimensiones del estilo de vida en los estudiantes de Tecnología Médica: la dimensión cuidado físico $p=0.000$; dimensión social 0.025 ; dimensión mental $p=0.000$; dimensión ético moral $\mathrm{p}=0.002$.

Conclusiones: Se demostró una influencia estadísticamente significativa en los cambios de actitud hacia la actividad física y el estilo de vida estudiantes de Tecnología Médica, es de suma importancia porque permite promover y proteger la salud de la comunidad universitaria, mejorando su calidad de vida.

Palabras claves: Actitud; actividad física; estilo de vida; estudiantes del área de la salud (Fuente: DeCS BIREME).

\section{Educational intervention program to promote the change of attitude toward physical activity and the improvement of the lifestyle of medical technology students in a private university}

\begin{abstract}
Objective: To determine the influence of an educational intervention program to promote attitude changes towards physical activity and lifestyle improvement in medical technology students of a private university.

Materials and methods: A quantitative, quasi-experimental, longitudinal, prospective study. The study sample included 94 students, divided into two groups: A (experimental group) and B (control group), which were created and designed by the author, and lasted 12 sessions in the Academic Semester 2016-II. Regarding the instruments used in the research, the Canopy test was used to measure the attitude towards physical activity; a 30-question questionnaire was applied to measure healthy lifestyles, resulting in a high-reliability value (Crombach's Alpha) of 0.95; and Student's t-test confirmed that there is a significant difference between pre-test and post-test averages.

Results: The educational intervention program influenced the indicator "perceived importance" with $p=0.000$. The attitude towards the indicator "practical activity" was $p=0.000$. As for lifestyle dimensions in medical technology students, physical care dimension reached $p=0.000$, social dimension $p=0.025$, mental dimension $p=0.000$, and moral ethical dimension $\mathrm{p}=0.002$.

Conclusions: Astatistically significant influence in attitude changes towards physical activity and lifestyle was demonstrated in medical technology students, which is extremely important because it enables the promotion and protection of the university community's health by improving its quality of life.
\end{abstract}

Keywords: Attitude; physical activity; life style; health occupations students. (Source: MeSH NLM).

1. Universidad Arzobispo Loayza. Lima, Perú.

a. Docente Universitario.

b. Doctor en Educación. 
Programa de intervención educativa para promover el cambio de actitud hacia la actividad física y la mejora del estilo de vida de los estudiantes de tecnología médica en una universidad privada

\section{INTRODUCCIÓN}

La universidad conlleva a un cambio importante en el individuo, influyendo en los estilos de vida, los estudiantes universitarios desde el ingreso a la universidad, existe un cambio en las actitudes y rutinas, que modifican hábitos y estilos de vida, relacionados con algunos factores académicos y conductuales típicos del quehacer universitario. Según avanzan en su formación académica van desarrollando conductas inadecuadas hacia su estilos de vida saludable, si no son atendidos de manera oportuna puede atentar en su salud, disminuir su capacidad de aprendizaje, padecer algún tipo de discapacidad y afectar su calidad de vida.

La actitud hacia la actividad física es importante para el desarrollo corporal e intelectual de la persona. La falta de esta puede acarrear varios problemas en la salud humana. Para lograr un cambio de actitud entre los estudiantes que no la practican, es importante buscar que se encuentren motivados ${ }^{(1)}$.

El profesional de la salud desempeña un rol como modelo social y sus hábitos personales influyen en el ejercicio profesional y la calidad de la prevención primaria que realiza. Una estrategia para mejorar su actitud es implantar un programa de promoción del ejercicio entre la población universitaria. De esta forma se previene que estos futuros profesionales desarrollen sobrepeso, obesidad y enfermedades crónicas asociadas a la baja actividad física (2).

Los jóvenes universitarios suelen realizar actividades con el fin de relajarse y descansar, compartir con la familia, los amigos o la pareja. También acuden con frecuencia a eventos culturales o artísticos. De esta manera, el tiempo de ocio también se identifica como un aspecto a considerar a la hora de promover estilos de vida saludables en esta población, particularmente en quienes trabajan mientras cursan los estudios ${ }^{(3)}$.

El tener un entorno social saludable es importante para lograr un cambio en el estilo de vida de los grupos de jóvenes profesionales. Por el contrario, uno que no lo es, afectará tanto la salud como la inserción social, creando una situación de riesgo y llegando a constituir un problema de salud pública por su magnitud y letalidad indirecta ${ }^{(4)}$.

Según el Ministerio de Salud (MINSA), sugiere la promoción de universidades saludables, con el propósito de consolidar a la universidad como un espacio en el que se cumplen las cinco funciones básicas de la promoción de la salud: desarrollar aptitudes personales para la salud, desarrollar entornos favorables, reforzar la acción comunitaria, reorientar los servicios de salud universitarios e impulsar políticas públicas de salud ${ }^{(5)}$.

La promoción para la salud en y desde el ámbito educativo parte de la visión integral, multidisciplinaria y transdisciplinaria del ser humano. Abordar la salud en el contexto de la educación universitaria trasciende la simple incorporación de conocimientos sanitarios para los estudiantes, surte efectos positivos en cuanto promoción, prevención y atención de la salud, aspectos que, a su vez, generan espacios que promuevan conductas y estilos de vida saludables ${ }^{(6)}$.

Resulta importante diseñar programas de intervención en los sujetos que tengan el deseo de modificar su comportamiento respecto a la práctica de actividad física así como su estilo de vida, ajustando las estrategias en función del estado de cambio en el que se sitúe cada individuo. Este hecho resulta relevante ya que en la etapa universitaria las personas pueden consolidar dicho estilo de vida, ejerciendo una gran influencia en los hábitos adquiridos en la etapa adulta ${ }^{(7)}$.

\section{MATERIALES Y MÉTODOS}

Es un estudio cuasi experimental. De corte longitudinal, siendo que las variables se midieron en dos tiempos diferentes. Se tuvo en consideración dos grupos de intervención: Grupo A (Grupo Caso) y Grupo B (Grupo Control), los datos fueron obtenidos y procesados en un software para análisis estadístico SPSS V. 23. Con los datos recolectados se procedió al análisis exploratorio con la finalidad de evaluar la normalidad de las variables con la prueba de Kolmogorov-Smirnoff y tomar la decisión de aplicar una prueba paramétrica (prueba $T$ Student para muestras independientes).

Presenta un enfoque cuantitativo, porque se analizaron datos numéricos, a través de la estadística, para verificar la hipótesis planteada y para dar solución a las preguntas de investigación. Se tomó el valor de significancia menor a $0.05(p<0.05)$.

Los grupos de la muestra han sido elegidos al azar, de forma que cada grupo de la población tuvo igual oportunidad de salir en la muestra a través de un muestreo no probabilístico a criterio del autor. Teniendo en consideración dos grupos: cuasi experimental: Estuvo conformado por 47 estudiantes de II ciclo de la Escuela de Tecnología Médica de la Universidad Privada San Juan Bautista Sede Lima Norte. En el grupo control: Está conformado por 47 estudiantes de II ciclo de la Escuela de Tecnología Médica de la Universidad Privada San Juan Bautista Sede Chorrillos. Para medir la actitud hacia la actividad física escala de actitudes hacia la actividad física y el deporte (E.A.F.D.), se elaboró un cuestionario titulado Cuestionario de estilos de vida saludable "El Universitario saludable", el cual su valor alcanzado por Alfa de Crombach fue de 0.995 , indicando que la confiabilidad del instrumento es altamente confiable. Se aplicó un programa de 12 sesiones, en el cual se abordó los temas de cuidado físico (alimentación saludable, consumo de sustancias tóxicas, actividad física, sueño y descanso); social (trabajo en equipo, relaciones interpersonales, actividades lúdicas y deportivas); mental (liderazgo), control de stress, toma de decisiones, autoestima); ético (valores en el ámbito deportivo), cada 
sesión tiene una duración de 60 minutos cada uno, se tuvo teniendo en consideración en cada sesión de clase se tuvo en consideración el siguientes fases: Fase de inicio: en donde el instructor presenta la información básica que será motivo de su exposición y se vale de diferentes recursos didácticos, donde se aprecia el contenido de la sesión de aprendizaje. El desarrollo del tema: el docente presenta la información detallada acerca del contenido de la sesión. Fase de cierre y/o reflexiva: Al finalizar la exposición, los participantes, reflexionan acerca del tema aprendido en la sesión de aprendizaje, el cual permitió desarrollar un cambio de actitud hacia la actividad física, mejorando su calidad de vida, asumiendo con responsabilidad y aplica en su haber cotidiano un estilo de vida saludable, valorando su salud.

\section{RESULTADOS}

Con respecto al puntaje de actitud hacia la actividad física y deportiva de los estudiantes de tecnología médica de acuerdo a la prueba paramétrica $\mathrm{T}$ Student para muestras independientes, tanto para el grupo de control y experimental, los estudiantes del grupo experimental obtuvieron mejores resultados en sus puntajes de actitud hacia la actividad física y deportiva $(63.9 \pm 9.7)$ después de la aplicación del programa de intervención educativa, respecto a los estudiantes del grupo de control (52.4 \pm 10.9). Existe una diferencia estadísticamente significativa $\mathrm{P}=0.000$ (Tabla 1).

Tabla 1. Actitud hacia la actividad física y deportiva de los estudiantes de Tecnología Médica de la Universidad San Juan Bautista - 2016

\begin{tabular}{|c|c|c|c|}
\hline \multirow[t]{2}{*}{ Estadístico } & \multicolumn{2}{|l|}{ Grupo } & \multirow[t]{2}{*}{ T Student } \\
\hline & Control $(n=47)$ & Experimental $(n=47)$ & \\
\hline \multicolumn{4}{|l|}{ Antes } \\
\hline Media & 51.2 & 51.7 & \multirow{2}{*}{$\begin{array}{l}\mathrm{t} \\
p=0.877\end{array}$} \\
\hline Desv. típ. & 13.2 & 15.9 & \\
\hline \multicolumn{4}{|l|}{ Después } \\
\hline Media & 52.4 & 63.9 & \multirow{2}{*}{$p=0.000^{\star * *}=-5.406$} \\
\hline Desv. típ. & 10.9 & 9.7 & \\
\hline
\end{tabular}

El rendimiento del puntaje del indicador: Actividad práctica de los estudiantes de tecnología médica, de acuerdo a la prueba paramétrica T Student para muestras independientes, tanto para el grupo de control y experimental. Los estudiantes del grupo experimental obtuvieron mejores resultados en sus puntajes de actitud hacia el indicador: Actividad práctica $(27.3 \pm 3.1)$ después de la aplicación del programa de intervención educativa, respecto a los estudiantes del grupo de control $(22.4 \pm 5.2)$. Se observa una diferencia significativa en los puntajes del rendimiento de actitud hacia la actividad física y deportiva de los estudiantes de tecnología médica (post test) entre los estudiantes del grupo de control y experimental, siendo éstos últimos los que obtuvieron mayores puntajes de rendimiento. Existe una diferencia estadísticamente significativa $\mathrm{P}=0.0000$ (Tabla 2).

Tabla 2. Actitud hacia el indicador: Actividad práctica de los estudiantes de Tecnología Médica de la Universidad San Juan Bautista - 2016

\begin{tabular}{|c|c|c|c|}
\hline \multirow[t]{2}{*}{ Estadístico } & \multicolumn{2}{|l|}{ Grupo } & \multirow[t]{2}{*}{ T Student } \\
\hline & Control $(n=47)$ & Experimental $(n=47)$ & \\
\hline \multicolumn{4}{|l|}{ Antes } \\
\hline Media & 21.9 & 21.5 & \multirow{2}{*}{$\begin{array}{l}\mathrm{T} \\
p=0.764\end{array}$} \\
\hline Desv. típ. & 6.2 & 6.8 & \\
\hline \multicolumn{4}{|l|}{ Después } \\
\hline Media & 22.4 & 27.3 & \multirow{2}{*}{$\begin{array}{l}\mathrm{t} \\
p=0.000^{* * *}\end{array}$} \\
\hline Desv. típ. & 5.2 & 3.1 & \\
\hline
\end{tabular}

El rendimiento del puntaje del indicador: importancia percibida de los estudiantes de tecnología médica es diferente al $95 \%$ de confiabilidad de acuerdo a la prueba paramétrica T Student para muestras independientes, tanto para el grupo de control y experimental según el post test, por lo que, los estudiantes del grupo experimental obtuvieron mejores resultados en sus puntajes de actitud hacia el indicador: importancia percibida (36.6 \pm 7.5$)$ después de la aplicación del programa de intervención educativa, respecto a los estudiantes del grupo de control $(29.9 \pm 6.4)$. 
Programa de intervención educativa para promover el cambio de actitud hacia la actividad física y la mejora del estilo de vida de los estudiantes de tecnología médica en una universidad privada

Se observa una diferencia significativa en los puntajes del rendimiento de actitud hacia la actividad física y deportiva de los estudiantes de tecnología médica (post test) entre los estudiantes del grupo de control y experimental, siendo éstos últimos los que obtuvieron mayores puntajes de rendimiento. Existe una diferencia estadísticamente significativa $\mathrm{p}=0.0000$ (Tabla 3).

Tabla 3. Actitud hacia el indicador: importancia percibida de los estudiantes de Tecnología Médica de la Universidad San Juan Bautista - 2016

\begin{tabular}{|c|c|c|c|}
\hline \multirow[t]{2}{*}{ Estadístico } & \multicolumn{2}{|l|}{ Grupo } & \multirow[t]{2}{*}{ T Student } \\
\hline & Control $(n=47)$ & Experimental $(n=47)$ & \\
\hline \multicolumn{4}{|l|}{ Antes } \\
\hline Media & 29.3 & 30.2 & \multirow{2}{*}{$\begin{array}{l}T \\
p=0.627\end{array}$} \\
\hline Desv. típ. & 7.5 & 9.7 & \\
\hline \multicolumn{4}{|l|}{ Después } \\
\hline Media & 29.9 & 36.6 & \multirow{2}{*}{$\begin{array}{l}\mathrm{t} \\
p=0.000^{* * *}\end{array}$} \\
\hline Desv. típ. & 6.4 & 7.5 & \\
\hline
\end{tabular}

El rendimiento del puntaje de la dimensión cuidado físico de los estudiantes de tecnología médica es diferente al $95 \%$ de confiabilidad de acuerdo a la prueba paramétrica T Student para muestras independientes, tanto para el grupo de control y experimental según el post test, Los estudiantes del grupo experimental obtuvieron mejores resultados en sus puntajes de la dimensión cuidado físico $(25.4 \pm 3.3)$ después de la aplicación del programa de intervención educativa, respecto a los estudiantes del grupo de control $(21.0 \pm 3.6)$, Existe una diferencia estadísticamente significativa $\mathrm{P}=0.000$ (Tabla 4).

Tabla 4. Dimensión cuidado físico de los estudiantes de Tecnología Médica de la Universidad San Juan Bautista - 2016

\begin{tabular}{|c|c|c|c|}
\hline \multirow[t]{2}{*}{ Estadístico } & \multicolumn{2}{|l|}{ Grupo } & \multirow[t]{2}{*}{ T Student } \\
\hline & Control $(n=47)$ & Experimental $(n=47)$ & \\
\hline \multicolumn{4}{|l|}{ Antes } \\
\hline Media & 21.5 & 21.6 & \multirow{2}{*}{$\begin{array}{l}T \\
p=0.871\end{array}$} \\
\hline Desv. típ. & 3.9 & 3.6 & \\
\hline \multicolumn{4}{|l|}{ Después } \\
\hline Media & 21.0 & 25.4 & \multirow{2}{*}{$\begin{array}{l}\mathrm{t} \\
p=0.000^{* * *}=-6.195\end{array}$} \\
\hline Desv. típ. & 3.6 & 3.3 & \\
\hline
\end{tabular}

El rendimiento del puntaje de la dimensión social de los estudiantes de tecnología médica es diferente al $95 \%$ de confiabilidad de acuerdo a la prueba paramétrica T Student para muestras independientes, tanto para el grupo de control y experimental según el post test. Los estudiantes del grupo experimental obtuvieron mejores resultados en sus puntajes de la dimensión social $(29.9 \pm 4.3)$ después de la aplicación del programa de intervención educativa, respecto a los estudiantes del grupo de control $(28.1 \pm 3.7)$. Existe una diferencia estadísticamente significativa $P=0.025$ (Tabla 5).

Tabla 5. Dimensión social de los estudiantes de Tecnología Médica de la Universidad San Juan Bautista - 2016

\begin{tabular}{|c|c|c|c|}
\hline \multirow[t]{2}{*}{ Estadístico } & \multicolumn{2}{|l|}{ Grupo } & \multirow[t]{2}{*}{ T Student } \\
\hline & Control $(n=47)$ & Experimental $(n=47)$ & \\
\hline \multicolumn{4}{|l|}{ Antes } \\
\hline Media & 27.7 & 25.2 & \multirow{2}{*}{$\begin{array}{l}T \quad=2.422 \\
p=0.017^{* \star *}\end{array}$} \\
\hline Desv. típ. & 4.8 & 5.4 & \\
\hline \multicolumn{4}{|l|}{ Después } \\
\hline Media & 28.1 & 29.9 & \multirow{2}{*}{$\begin{array}{l}\mathrm{t}=-2.273 \\
p=0.025^{\star * *}\end{array}$} \\
\hline Desv. típ. & 3.7 & 4.3 & \\
\hline
\end{tabular}


El rendimiento del puntaje de la dimensión mental de los estudiantes de tecnología médica es diferente al $95 \%$ de confiabilidad de acuerdo a la prueba paramétrica T Student para muestras independientes, tanto para el grupo de control y experimental según el post test, por lo que, los estudiantes del grupo experimental obtuvieron mejores resultados en sus puntajes de la dimensión mental $(19.8 \pm 3.7)$ después de la aplicación del programa de intervención educativa, respecto a los estudiantes del grupo de control $(16.4 \pm 4.7)$. Existe una diferencia estadísticamente significativa $P=0.000$ (Tabla 6).

Tabla 6. Dimensión mental de los estudiantes de Tecnología Médica de la Universidad San Juan Bautista - 2016

\begin{tabular}{lccll}
\hline Estadístico & $\begin{array}{l}\text { Grupo } \\
\text { Control }(n=47)\end{array}$ & Experimental $(n=47)$ & & T Student \\
\hline $\begin{array}{l}\text { Antes } \\
\text { Media }\end{array}$ & 16.4 & 16.0 & T & $=0.511$ \\
Desv. típ. & 3.8 & 4.7 & $p=0.611$ & \\
\hline $\begin{array}{l}\text { Después } \\
\text { Media }\end{array}$ & 16.4 & 19.8 & $\mathrm{t}$ & $p=0.000^{* \star}=-3.908$ \\
Desv. típ. & 4.7 & 3.7 & &
\end{tabular}

El rendimiento del puntaje de la dimensión ético - moral de los estudiantes de tecnología médica es diferente al $95 \%$ de confiabilidad de acuerdo a la prueba paramétrica T Student para muestras independientes, tanto para el grupo de control y experimental según el postest. Los estudiantes del grupo experimental obtuvieron mejores resultados en sus puntajes de la dimensión ético - moral $(32.5 \pm 4.4)$ después de la aplicación del programa de intervención educativa, respecto a los estudiantes del grupo de control $(29.7 \pm 4.3)$. Existe una diferencia estadísticamente significativa $P=0.002$ (Tabla 7).

Tabla 7. Dimensión ético - moral de los estudiantes de Tecnología Médica de la Universidad San Juan Bautista - 2016

\begin{tabular}{|c|c|c|c|c|}
\hline \multirow[t]{2}{*}{ Estadístico } & \multicolumn{2}{|l|}{ Grupo } & \multirow{2}{*}{\multicolumn{2}{|c|}{ T Student }} \\
\hline & Control $(n=47)$ & Experimental $(n=47)$ & & \\
\hline \multicolumn{5}{|l|}{ Antes } \\
\hline Media & 29.7 & 29.1 & \multirow{2}{*}{$\begin{array}{l}\mathrm{T} \\
p=0.499\end{array}$} & \multirow[t]{2}{*}{$=0.679$} \\
\hline Desv. típ. & 4.0 & 5.3 & & \\
\hline \multicolumn{5}{|l|}{ Después } \\
\hline Media & 29.7 & 32.5 & \multirow{2}{*}{\multicolumn{2}{|c|}{$\begin{array}{l}\mathrm{t} \\
p=0.002^{\star \star *}=-3.151\end{array}$}} \\
\hline Desv. típ. & 4.3 & 4.4 & & \\
\hline
\end{tabular}

\section{DISCUSIÓN}

Se dará a conocer el análisis de los resultados hallados, contrastados con los de otros estudios realizados, los cuales se relacionan de manera directa e indirecta con las variables y muestra de investigación y opinión propia del autor. El estudio a pesar que, contó con los recursos humanos y materiales, y que la disponibilidad de los estudiantes de Tecnología estuvo basado bajo los principios fundamentales de la bioética (justicia, autonomía, no maleficencia y beneficencia), una limitación de importancia fue el cálculo de la muestra no probabilística, la que no permite generalizar los resultados obtenidos, asimismo se aplicó solo en los estudiantes de II Ciclo y no en todos los estudiantes de Tecnología Médica de la UPSJB.

El programa de intervención educativa influye en promover el cambio de actitud hacia la actividad física $(p=0.000)$, en la revisión bibliográfica, similar a lo reportado por Pérez IJ (8); Pérez I (9) el cambio de actitud hacia la actividad física está íntimamente ligada al tipo de estilo de vida que lleve el estudiante universitario, esto conlleva a la mejora del aprendizaje y la calidad de vida, estos tipos de programas preventivos es importante en las poblaciones infantes y juveniles, con o sin alguna enfermedad, modificando sus patrones de conducta, resaltando que las funciones 
Programa de intervención educativa para promover el cambio de actitud hacia la actividad física y la mejora del estilo de vida de los estudiantes de tecnología médica en una universidad privada

fisiológicas y cognitivas. Es importante la aplicación de este tipo de programas en la población universitaria porque permite modificar sus patrones de conducta para que puedan tener una calidad de vida óptima.

En cuanto a la mejora la del estilo de vida de los estudiantes de tecnología médica de la universidad San Juan Bautista se ha encontrado una diferencia estadísticamente significativa $(p=0.000)$, existen autores como los de Jiménez J (2); Quilla R ${ }^{(10)}$; Guevara DM (11); Espinoza LR ${ }^{(12)}$; Matute EL (13); Pérez A ${ }^{(14)}$. Los programas de intervención educativa en promover estilos de vida saludable es de vital importancia en la población adulta joven en el desarrollo de una adecuada educación integral, el desarrollo personal y prevención de enfermedades en el futuro profesional. Se recomienda implementar estrategias en todas las personas de todas las edades un estilo de vida saludable que permita un óptimo desarrollo físico y cognitivo.

En lo que respecta a la dimensión cuidado físico, se ha encontrado una diferencia estadísticamente significativa $(p=0.00)$, estudios similares como los de Veramendi $N{ }^{(15)}$; Quilla R (10); Shahnazi H (16); Madipour N (17); Guevara DM (11); Pérez $\mathrm{A}^{(14)}$; el cuidado físico es una condición en que la persona, toma conciencia acerca de salud corporal, en la prevención de riesgos que podrían afectar su funcionalidad. Es necesario elegir una alimentación que aporte todos los nutrientes esenciales y la energía que toda persona necesita para mantenerse en un óptimo estado de salud, evitando el consumo de sustancias tóxicas, realizar actividad física, tener un descanso corporal, para que el estudiante tenga un óptimo rendimiento físico cognitivo.

Con respecto a la dimensión social, se observó que existe una diferencia estadísticamente significativa $p=0.025$, estudios similares como los de Mahdipour $N$ (17); Limachi $K{ }^{(18)}$. El apoyo social es una ayuda emocional, así como el estímulo de otros grupos tales como los vecinos, familia, amigos y líderes de la comunidad quienes harán posible practicar comportamientos que conduzcan a estilos de vida saludables.

En cuanto a la dimensión mental, existe una relación estadísticamente significativa, $\quad(p=0.000), \quad$ estudios similares como los de Quilla R (10); Mahdipour N (16); Guevara DM (11); Pérez A ${ }^{(14)}$. Los estudiantes universitarios son vulnerable a diversos problemas en su salud mental siendo que desde su ingreso, están sometidos a factores que contribuirán a volverlos vulnerables según avanzan en su formación profesional, como adaptarse a la vida universitaria, los horarios y las exigencias, las condiciones económicas, el sentimiento de alejamiento familiar, el fracaso, que pueden generar síntomas depresivos Rosas $M$ (19).

Ese tipo de programa proporciona bienestar emocional, desarrollando actitudes positivas, reduce los factores estresantes. Así mismo, permite lograr confianza, fortaleza y capacidad para enfrentar nuevos retos.

En la dimensión ético-moral, se ha observado una diferencia estadísticamente significativa, la actitud ético moral es importante en el cambio de actitud hacia la actividad física como en los diferentes estilos de vida saludable en el estudiante universitario, partiendo del respeto a las normas, creará en el estudiante cambios en su actitud , el compromiso de poder ejecutar diariamente sin presión u obligación, la solidaridad permitirá mejorar su sociabilización con sus demás compañeros de clase permitiendo despertar el interés hacia la actividad física .

En conclusión, el programa de intervención educativa influyó positivamente en el cambio de actitud hacia la actividad física y los estilos de vida saludable en los estudiantes de II ciclo de Tecnología Médica de la UPSJB. Este tipo de programas es fundamental porque impulsa una alternativa en el cuidado de salud integral del estudiante universitario, siendo que el estudiante de pregrado no solo debe estar capacitado bajos las competencias académicas propias de su carrera profesional sino debe hallarse física y mentalmente preparado para poder desempeñar las funciones que se le van hacer encomendadas en su haber profesional.

\section{REFERENCIAS BIBLIOGRÁFICAS}

1. Alias A, Aguilar JM, Hernandez-Rodriguez Al. Motivaciones de los estudiantes Universitarios ante la práctica de actividad físico-deportiva de tiempo libre. Las actividades náuticas. Psychol Soc Educ. 2016;8(3):229-242. Disponible en: http:// repositorio.ual.es/handle/10835/4782

2. Jiménez-Díaz J, Morera-Castro $M$. Desempeño motor y hábitos de actividad física en estudiantes universitarios en Costa Rica. Rev MHSalud. 2016;12(2):1-14. Disponible http://www. redalyc.org/html/2370/237043581002/

3. Varela-Arévalo MT, Ochoa-Muñoz AF, Tovar-Cuevas JR. Tipologías de estilos de vida en jóvenes universitarios. Rev Univ Salud. 2016;18(2):246-56. Disponible: http: / /www.scielo.org.co/scielo.php?script=sci_ abstract\&pid=S0124-71072016000200006

4. Ledesma LEB, Cosain EIH, Martínez SE, Cosain YBH, Preza MAG, Valles JAR, et al. Explorar el estilo de vida prevaleciente en estudiantes del área de la salud. Rev Electr Med Salud y Soc. 2015;6(1):51-64. Disponible en: http://cienciasdelasaluduv. com/revistas/index. php/mss/article/view/191

5. Ministerio de Salud [sede Web]. 2015 [acceso 12 de Marzo de 2017]. Disponible en: http://bvs.minsa.gob.pe/local/ MINSA/3396.pdf .

6. Malpica A, Dugarte A. Salud Integral y Calidad de vida de los estudiantes de Ciencias de la Educaciòn. Universidad de Carabobo Valencia Venezuela. Rev Dialógica. 2017;12(2):4979. Disponible en: http://revistas.upel.edu.ve/index.php/ dialogica/article/view/4458 
7. Práxedes A, Sevil J, Moreno A, Del Villar F, García-González L. Niveles de actividad física en estudiantes universitarios: diferencias en función del género, la edad y los estados de cambio. Rev Iberoam Psicol Ejerc. deporte. 2016;11(1): 123-132. Disponible en: http://www.redalyc.org/ html/3111/311143051014/

8. Pérez IJ, Delgado Fernández M. Modificación de las actitudes del alumnado de secundaria hacia la práctica de actividad. Rev Psicol Deport. 2003;12(2):0165-179. Disponible en: https://ddd.uab.cat/record/63077

9. Pérez I, Delgado M. Mejora de los conocimientos, procedimientos y actitudes del alumnado de secundaria tras un programa de intervención en educación física para la salud. Motricidad. Eur J Hum Mov. 2007;18: 61-77. Disponible en: http://www.redalyc.org/html/2742/274220374004/

10. Quilla R, Maquera E, León DL. Eficacia del programa "adolescencia saludable" para mejorar estilo de vida en estudiantes del 4to año de educación secundaria de un colegio privado confesional. Apunt Univ. 2016; 6(1), 79-86. Disponible en: http://revistascientificas.upeu.edu.pe/index. $\mathrm{php} /$ ra_universitarios/article/view/505

11. Guevara DM, Henríquez MEG. Efectividad del Programa Educativo Aprendiendo Estilos de vida. Rev In Crescendo Cienc Salud, 2014; 1(2), 243-250. Disponible en: http:// revistas.uladech.edu.pe/index.php/increscendo-salud/ article/view/342/234

12. Espinoza LR. Efecto de una estrategia de intervención educativa en el desarrollo de competencias saludables y estilo de vida en jóvenes universitarios chilenos [Tesis Doctoral]. Universidad de Granada, Granada, España; 2010.

13. Matute EL, Sevillano Z, Torres R, Salazar N, Díaz C, Vásquez PM, et al. Programa educativo:"Estilos de vida saludable y su impacto en pobladores del pueblo joven Túpac Amaru. lquitos 2007-2008". Rev Conoc Amaz. 2015;1(1):103-8. Disponible en: http://revistas.unapiquitos.edu.pe/index. $\mathrm{php} /$ Conocimientoamazonico/article/view/13/20

14. Pérez A. Rojas A. Diseño y Evaluación de un Programa para desarrollar estilos de vida promotores de salud [Tesis Licenciatura en Psicología]. Benemèrica Universidad Autònoma de Puebla, Mèxico;2007.

15. Veramiendi $\mathrm{N}$. Intervención de estrategias de enseñanza en estilos de vida saludables de las amas de casa, localidad de Pillcomarca-Huánuco 2012. Rev Educ Desarr Social. 2013; 7(1), 63-70. Disponible en: https://search.proquest.com/ openview/f39b55c084665a327f12e1d57d8eb599/1?pqorigsite $=$ gscholar\&cbl $=2042933$

16. Shahnazi H, Koon PB, Talib RA, Lubis SH, Dashti MG, Khatooni
E, et al. Can the BASNEF model help to develop selfadministered healthy behavior in Iranian youth? Iran Red Crescent Med J. 2016;18(3). Disponible en: https://www. ncbi.nlm.nih.gov/pmc/articles/PMC4879761/

17. Mahdipour N, Shahnazi H, Hassanzadeh A, Sharifirad G. The effect of educational intervention on health promoting lifestyle: Focusing on middle-aged women. J Educ Health Promot 2015;4: 51. Disponible en: https://www.ncbi.nlm.nih. gov/pmc/articles/PMC4579766/

18. Limachi KEM. Efectividad del programa educativo "Ejercicio es Vida" en los conocimientos, actitudes y prácticas sobre el ejercicio físico que poseen los estudiantes del $3^{\circ}$ año de enfermería de la Universidad Peruana Unión, abril-junio 2010. Rev Cient Cienc Salud. 2011;4(1):50-60. Disponible en: http://revistascientificas.upeu.edu.pe/index.php/rc_salud/ article/view/164/654

19. Rosas M, Yampufé M, López M, Carlos G, Sotil de Pacheco A. Niveles de depresión en estudiantes de Tecnología Médica. An Fac Med. 2011; 72 (3), 183-186. Disponible en: http://www.scielo.org.pe/scielo.php?pid=S1025$55832011000300005 \&$ script=sci_arttext\&tlng=en

Fuentes de financiamiento:

Este artículo ha sido financiado por el autor.

Conflictos de interés:

El autor declara no tener ningún conflicto de interés.

Correspondencia:

Cristhian Santiago Bazán

Dirección: Jr. Tiahuanaco 1493 Urb. Zárate. S.J. L

Teléfono: 941703104

Correo electrónico: cristhiansantiagob@gmail.com

Recibido: 28 de agosto de 2017

Evaluado: 30 de agosto de 2017

Aprobado: 06 de enero de 2018

(c) La revista. Publicado por Universidad de San Martín de Porres, Perú. (c) ву Licencia de Creative Commons Artículo en acceso abierto bajo términos de Licencia Creative Commons Atribución 4.0 Internacional. (http://creativecommons.org/licenses/by/4.0/)

\section{ORCID iDs}

Cristhian Santiago Bazán

https: / / orcid.org/0000-0001-9073-4785 